\title{
Archéopages
}

Archéopages

Archéologie et société

42 | 04-07/2015

Construire en terre crue

\section{Des carrières-refuges pendant la Bataille de Normandie}

Laurent Dujardin et Cyril Marcigny

\section{OpenEdition}

1 Journals

Édition électronique

URL : https://journals.openedition.org/archeopages/1344

DOI : 10.4000/archeopages. 1344

ISSN : 2269-9872

Éditeur

INRAP - Institut national de recherches archéologiques préventives

Édition imprimée

Date de publication : 1 mars 2016

Pagination : 138-139

ISSN : 1622-8545

\section{Référence électronique}

Laurent Dujardin et Cyril Marcigny, « Des carrières-refuges pendant la Bataille de Normandie »,

Archéopages [En ligne], 42 | 04-07/2015, mis en ligne le 01 juillet 2017, consulté le 03 juin 2021. URL http://journals.openedition.org/archeopages/1344; DOI : https://doi.org/10.4000/archeopages.1344 


\section{Des carrières-refuges pendant la Bataille de Normandie}

\author{
Laurent Dujardin \\ UMR 6273-CRAHAM \\ Cyril Marcigny \\ Inrap, UMR 6566-CReAAH \\ Responsables d'opération
}

Site

Carrière Saingt

Fleury-sur-Orne

Calvados

Date

Mai 2014

Équipe

Étude du site et des mobiliers

Sylvain Mazet

Inrap

Pierre Mazure

bénévole

Christophe Prime

Mémorial de Caen

Relevé 3D

Laurent Carozza

Albane Burens

CNRS

Topographie

Laurent Vipard

Inrap

Pierre Grussenmeyer

Samuel Guillemin

INSA

Étude historique

Vincent Carpentier

Inrap

1

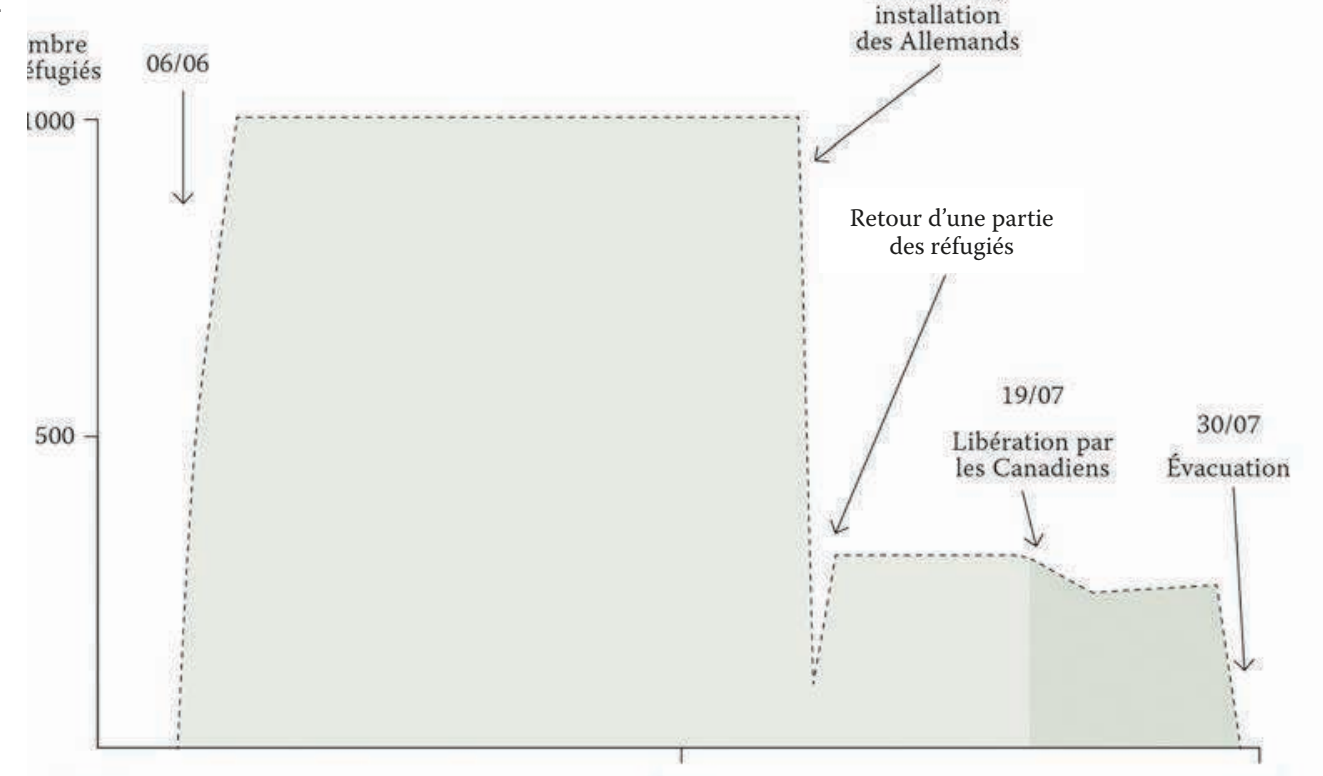

$01 / 06$
Après le Débarquement allié en 1944, entre le 6 juin et le 30 juillet, lors de la Bataille de Normandie, quasi un millier d'habitants de Caen et de Fleury-sur-Orne ont trouvé refuge dans une carrière souterraine de pierre à bâtir creusée au milieu du XIX ${ }^{\mathrm{e}}$ siècle, près de la route d'Harcourt [ill. 1]. Son accès était connu et aisé car elle avait été utilisée par une brasserie entre les deux guerres mondiales. À l'issue des combats, la carrière est close, jusqu'aux années 1980. À l'époque, les premières observations des traces des réfugiés commencent sous la conduite d'historiens et de spéléologues. Dans les années 1990 et 2000 est réalisée une première couverture photographique (par D. Butaeye et L. Dujardin), qui a permis de montrer l'importance des traces archéologiques encore visibles et de commencer un relevé des nombreux objets en surface. Cette étude a été complétée en 2008 et 2009 par le recueil de témoignages oraux et photographiques [ill. 2] (Dujardin, 1998; Dujardin et Butaeye, 2008).

Lorsque débute le chantier de lotissement dans ce quartier de la périphérie caennaise, en 2005 , le promoteur a été sensibilisé à la richesse patrimoniale de la carrière par les spéléologues et les ouvriers eurent pour consigne de ne pas perturber les zones archéologiques. Bien que les travaux aient été conduits sans suivi archéologique, les dégâts ont ainsi été minimisés par rapport à ceux infligés à d'autres sites souterrains médiévaux, modernes ou contemporains de la région :

l'accès principal de la carrière a été comblé de matériaux de construction et quelques parties de la carrièrerefuge ont été détruites par le passage d'un engin chenillé. Il semble que le site n'ait pas reçu par la suite de visites de pillards. En dehors de ces parties bouleversées ou détruites par le chantier, il n'y a donc pas eu d'atteinte notable aux traces archéologiques. Les observations menées sur presque trois décennies font penser que ce qui est encore visible actuellement l'aurait été, à peu de différence près, en 194.4

Aujourd'hui, les traces les plus visibles occupent une aire de plusieurs centaines de mètres carrés ; des parties importantes de la carrière semblent ne pas avoir servi, probablement en raison de leur trop grande humidité. D'autres zones ne recèlent que peu de traces mais des investigations fines restent nécessaires. Une cuisine collective avait été mise en place [ill. 3]. Des aires damées sont réparties autour de chemins, et des tiges métalliques, qui ont servi de supports à des étagères ou pour retenir des draps afin de garantir une intimité minimum, sont plantées dans les piliers de la carrière [ill. 4].

Véritable conservatoire archéologique, le site de la carrière Saingt offre de nombreux champs d'investigations, que ce soit sur les modalités d'occupation des lieux au cours des événements de 1944, les comportements sociaux en milieu confiné ou l'archéologie industrielle. Dans le but de préserver l'intégrité de ce site, les méthodes d'acquisition de données utilisées à Fleury-sur-Orne sont non destructives et favorisent le recours à des techniques d'enregistrement et de prise de mesures sans contact (balayage laser et photogrammétrie) [ill. 5]. Ces relevés permettent non seulement de produire des données $2 \mathrm{D}$ (coupes ou élévations), mais aussi et surtout des modèles $3 \mathrm{D}$ calculés à partir des nuages de points, des vues en perspective photoréalistes et la possibilité de naviguer dans les modèles $3 \mathrm{D}$ avec des outils dédiés à la visualisation de l'environnement (Grussenmeyer et al., 2012).

Ce sont ces méthodes, impliquant différentes techniques basées sur une variété de scanners à balayage et d'appareils photos terrestres (de type réflex numérique) ainsi que d'autres systèmes d'imagerie spatiale, que nous avons testés en 2014. Dans les prochaines années, le modèle $3 \mathrm{D}$ géométrique et photoréaliste de l'ensemble des éléments structurels de la carrière sera ainsi réalisé. Les objets archéologiques seront également géoréférencés et numérisés pour être associés au modèle numérique de terrain (MNT) global. Les modèles sont ainsi conçus comme des outils de recherche communs, utilisables par tous les partenaires de l'équipe scientifique, et pouvant servir d'outil de documentation et de visualisation.

Cevaste programme de recherche a donc principalement une double ambition. Celle de proposer une archéologie de l'enfermement confrontée aux sources écrites et orales, livrant un référentiel pour examiner a posteriori des sites plus anciens (grottes-refuges de la protohistoire ancienne, par exemple), offrant ainsi de nouvelles clefs d'interprétation. Et celle de développer des outils de relevés et d'analyse performants pour l'examen de sols d'occupation (taphonomie, techniques $3 \mathrm{D}$...).

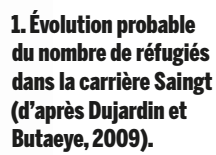



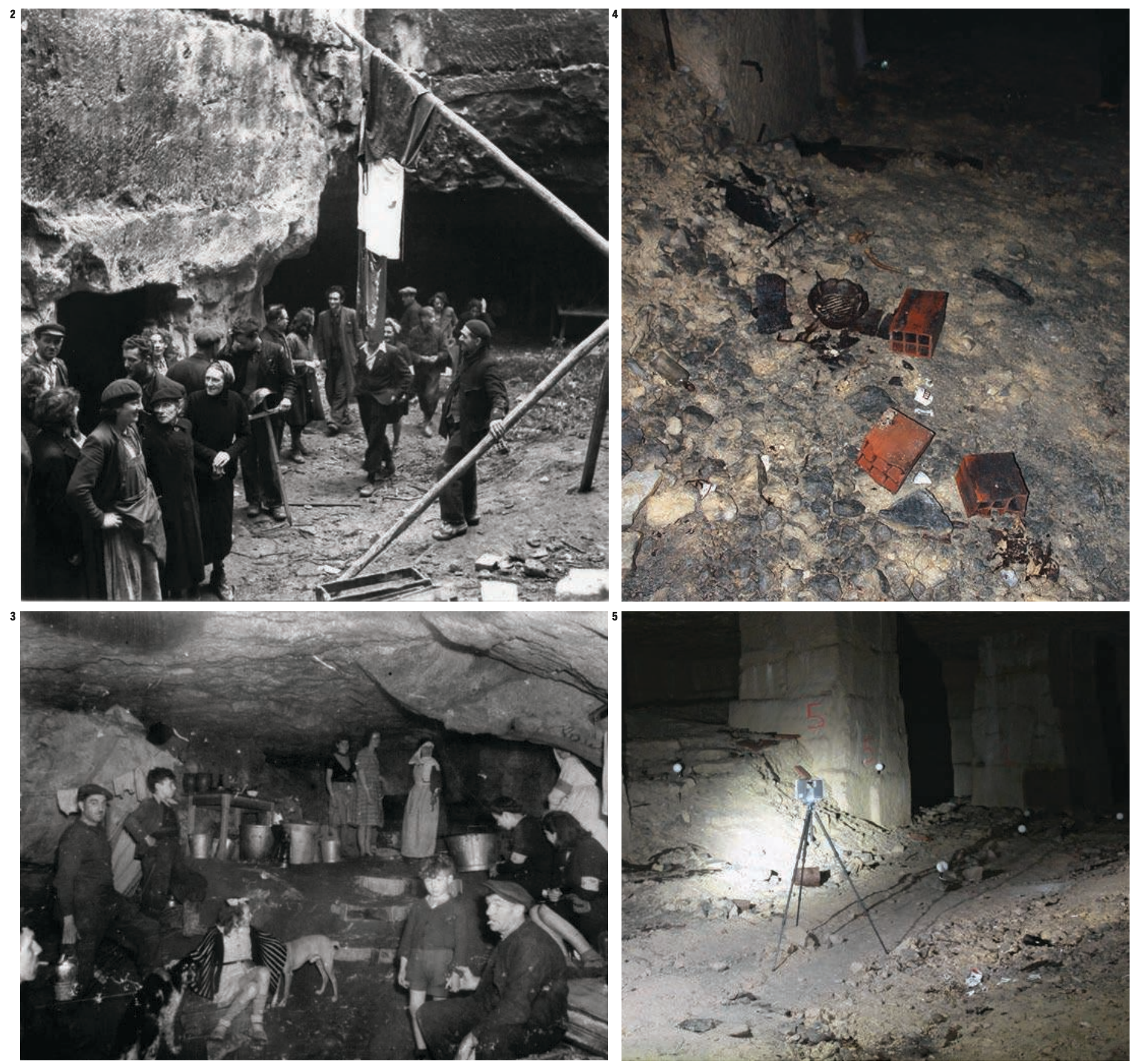

2. Les réfugiés de la carrière Saingt lors de leur libération en juillet 1944. Un drapeau français de fortune composé de vêtements assemblés

trône au-dessus de la tirée, accès initial de la carrière

3. Scène de vie dans

la carrière des Coteaux

à Fleury-sur-Orne.

4. Quelques vestiges visibles

à la surface des sols

d'occupation.

\section{Relevé au scan de la}

volumétrie générale d'un des

espaces de Fleury-sur-Orne ;

les espaces de vie et les

vestiges gisent aux pieds

des piliers de la carrière.
Référence bibliographique

Grussenmeyer P., Burens A., Moisan E., Guillemin S., Carozza L.

Bourrillon R., Petrognani

2012, «3D Multi-scale Scanning of the

Archaeological Cave "Les Fraux" in

Dordogne (France) », in IONNADEs M. et al. (éd.), Progress in Cultural Heritage Preservation, $4^{\text {th }}$ International Conference, EuroMed 2012, Lecture Notes in Computer Science, 7616 p. 388-395.

Dujardin L., Butaeye D., 2009, Les réfugiés dans les carrières pendant la bataille de Caen, juin-juillet 1944, Rennes, éd. Ouest-France, 143 p. Dujardin L., 1998, Carrières de pierre en Normandie. Contribution à létude historique et archéologique des carrières de pierre à bâtir à Caen (Calvados) et en Normandie aux époques médiévale et moderne, Thèse de doctorat d'Histoire et d'Archéologie de l'Université de Caen. 